\title{
Cyclic Cosmology and Holographic Entanglement Entropy
}

\author{
P.H. Frampton* \\ Dipartimento di Matematica e Fisica "Ennio De Giorgi", \\ Universita del Salento and INFN-Lecce, \\ Via Arnesano, 73100 Lecce, Italy. \\ *E-mail: paul.h.frampton@gmail.com
}

Keywords: Turnaround; Entropy; Bounce; Connectivity.

\section{Introduction}

Cyclic cosmology with an infinite number of cycles each of which includes expansion, turnaround, contraction and bounce, is an appealing theory, and one of the most recent attempts at a solution was in 2007 by Baum and me $e^{1}$. As emphasized first by Tolman, entropy and the second law of thermodynamics provide a significant challenge to implementing a cyclic theory $\stackrel{2}{2}$.

The approach $\underline{1}$ was based on the so-called Come Back Empty (CBE) principle which chooses the turnaround as the point in the cycles where entropy is jettisoned. A successful universe must be selected with zero entropy at the turnaround to begin an adiabatic contraction to the bounce while empty of all matter including black holes. In this article we provide a better understanding of CBE based on the holographic principle. In particular, the notion of entanglement entropy and the related question of spacetime connectivity can put the CBE model on a quantum theoretical basis.

Although the holographic principle was well-known at that time, its relationship to cyclic cosmology was unclear. Even how it could help reconcile general relativity with quantum mechanics was less developed. In the AdS/CFT correspondence, it has been argued that the dynamics of spacetime on the AdS side, including the connectivity of spacetime, is related to quantum entanglement of disconnected classi-cal theories on the CFT side. This idea was introduced by Van Raamsdonk in an essay submitted to the Gravity Research Foundation? . Considerations of the entropy of the universe have been discussed also by E. Verlinde ${ }^{3}$.

Before discussing the impact on cyclic cosmology which is our present subject, let us briefly discuss how this line of reasoning changes the marriage of general relativity with quantum mechanics. For several decades it had been tacitly assumed that there exists a quantum gravity theory whose classical limit is general relativity. Now it appears that whether or not such a theory exists may not be the best question to ask. Classical general relativity which describes the dynamics of spacetime may instead 
arise from consistency conditions on the quantum entanglement of conformal field theories which are at the holographic boundary.

Subsequent work has been mainly on time-independent spacetimes. However, we shall show that for the the time-dependent CBE model of cyclic cosmology use of holographic quantum entanglement entropy makes possible a better understanding of entropy at the turnaround. First we briefly review the CBE model.

The best way to discuss the CBE model is by using a language where the spacetime occupied by the universe is divided into two subregions denoted by introverse and extroverse respectively which we must define carefully because it will be the entanglement entropy between these two subregions which will play a central rôle in the cyclicity. The present visible universe at $t=t_{0}$ is the present introverse and its comoving radius is $R_{\text {int }}\left(t_{0}\right)=44 G l y$. A more useful time to consider than the present $t_{0}=13.8 G y$ is the time $t=t_{D E}$ when the dark energy began to dominate over the matter component. This occurred at $t_{D E}=9.8 G y$ at which time the extroverse is identified with the introverse.

After $t=t_{D E}$, the extroverse expands exponentially and become larger, eventually very much larger, than the introverse.

At $t=t_{D E}$ we have

$$
R_{e x t}\left(t_{D E}\right)=R_{\text {int }}\left(t_{D E}\right)=39 G l y
$$

at a time when the scale factor, normalized to $a\left(t_{0}\right)=1$, was $a\left(t_{D E}\right)=0.75$.

The extroverse expands exponentially for $t_{D E}<t<t_{T}$, where $t_{T}$ is the turnaround time calculated to be $t_{T}=1.3 \mathrm{Ty}$. The extroverse comoving radius during this expansion is given by

$$
R_{e x t}(t)=(39 G l y) \exp \left[\frac{t-9.8 G y}{13.8 G y}\right]
$$

Two interesting values at $t=t_{0}$ and $t=t_{T}$ respectively are

$$
R_{\text {ext }}\left(t_{0}\right)=52 G l y \quad \text { and } \quad R_{\text {ext }}\left(t_{T}\right)=1.5 \times 10^{42} \text { Gly }
$$

The introverse expands more gradually for $t_{D E}<t<t_{T}$, being limited by the speed of light in its definition. The introverse comoving radius for the expansion era is given in terms of $a(t)=\exp [(t-13.8 G y) /(13.8 G y)]$ by 


$$
\begin{aligned}
R_{\text {int }}(t)= & 39 G l y+c \int_{t_{D E}}^{t} d t a(t)^{-1} \\
= & 39 G l y+ \\
& (13.8 G l y)[1-\exp \{-(t-9.8 G y) /(13.8 G y)\}] \\
= & 44 G l y+ \\
& (13.8 G l y)[1-\exp \{-(t-13.8 G y) /(13.8 G y)\}]
\end{aligned}
$$

From Eq. (4) the values of $R_{\text {inv }}(t)$ at $t=t_{0}$ and $t=t_{T}$ are

$$
R_{\text {int }}\left(t_{0}\right)=44 G l y \quad \text { and } \quad R_{\text {inv }}\left(t_{T}\right)=58 G l y
$$

The behaviour of $R_{e x t}(t)$ and $R_{i n v}(t)$ calculated in (3) and (5) respectively assumes that the dark energy is accurately described by a cosmological constant. The results for $R_{e x t}\left(t_{0}\right)$ and $R_{i n v}\left(t_{0}\right)$ show that the present extroverse is already $60 \%$ larger in volume than the introverse implying that hundreds of billions of galaxies have exited the introverse since the onset of exponential expansion.

We have previously used a precise value for the turnaround time $t_{T}=1.3 T y$ without further comment so we provide a few more words. For infinite cyclicity, it is necessary to impose a matching condition on the contraction scale factor with the scale factor of the previous expansion. Since the contraction has a radiation-dominated behaviour $\propto \sqrt{t}$ the matching is conveniently made at the onset of matter domination during expansion when $t=t_{m}=47 \mathrm{ky}$. This leads to $t_{T}=1.3 T y$ for the turnarounds time. We note that this could not be derived in the BF model $\underline{\underline{1}}$ because there the equation of state for the dark energy was assumed to be $\omega<-1$ but cyclicity is now known to require $\omega=-1$ precisely.

We shall improve the original version of Comes Back Empty (CBE) with a new understanding based on quantum entanglement. Although quantum fluctuations as precursors of cosmic structure formation are well-known, to our knowledge this is the first application of quantum computation and quantum information, in par- ticular quantum entanglement, to theoretical cosmology. To see that it is an improvement, let us first describe the original CBE model.

In the original derivation which used phantom dark energy with $\omega<-1$, a modified Friedmann equation in the form

$$
\left(\frac{\dot{a}}{a}\right)^{2}=\frac{8 \pi G}{3}\left[\rho-\frac{\rho^{2}}{\rho_{c r}}\right]
$$

was used where $\rho_{c r}$ is a constant. Such a modified Friedmann equation was derived from string theory. This led to a bounce when $\rho=\rho_{c r}$ at a time $<10^{-27} s$ before 
the Big Rip. The scale factor deflated dramatically to $\hat{a}\left(t_{T}\right)=f a\left(t_{T}\right)$ where $f<$ $10^{-28}$. All bound states become unbound and soon afterwards become causally disconnected. The critical density is $\rho_{c r}=\eta \rho_{\mathrm{H}_{2} \mathrm{O}}$ with $\eta$ between $10^{31}$ and $10^{87}$, safely below the Planck value $\eta=10^{104}$. A given patch is chosen so that it is empty of all matter including black holes, and contains only dark energy. This causal patch contracts adiabatically to a bounce with zero entropy.

In the present derivation, the dark energy is described by a cosmological constant with $\omega=-1$ so that we must use the unmodified Friedmann equation

$$
\left(\frac{\dot{a}}{a}\right)^{2}=\frac{8 \pi G}{3} \rho
$$

at almost all times except times extremely close to the turnaround or the bounce.

Now we turn to the rôle of quantum entanglement. This begins from the holographic principle and its realization in the AdS/CFT duality. One previous use of quantum mechanics in cosmology has been the idea that quantum fluctuations of the inflaton field during inflation are amplified to seed large scale structure formation. Here we suggest that quantum entanglement and its relationship with spacetime connectivity play an comparably dramatic rôle by elucidating the CBE cyclic cosmology model at turnaround.

In the AdS/CFT correspondence, if we consider two non-interacting identical copies $C F T_{A}$ and $C F T_{B}$, a state of the system can in general be written

$$
|\Psi>=| \Psi>_{A} \otimes \mid \Psi>_{B} .
$$

The CFTs are each dual to separate asymptotically AdS spacetimes so that the direct product (8) is dual to two spacetimes which are disconnected.

As a quantum state we are free to consider a superposition of states. Let the energy eigenstates be $\mid E_{k}>$ for one CFT and consider the quantum superposition

$$
\left|\Psi(\beta)>=\Sigma_{k} e^{-\frac{\beta}{2} E_{k}}\right| E_{k}>\otimes \mid E_{k}>
$$

which can be shown, in general, to be dual to a single connected spacetime. Starting with $\mid \Psi>$ we deduce that the denstiy matrix for $C F T_{B}$ is

$$
\operatorname{Tr}(|\Psi><\Psi|)=\Sigma_{k} e^{-\beta E_{k}}\left|E_{k}><E_{k}\right|=\rho_{T}
$$

which is a thermal density matrix. so the quantum superposition of two disconnected spacetimes is identified with a classical connected spacetime. This leads to the fascinating idea that classical spacetime connectivity arises by quantum entangling the degrees of freedom in two components 
which is a central idea in what follows.

Proceeding in the opposite direction, let us consider one CFT on a sphere and divide the sphere initially into two hemispheres A and B with Hilbert space $\mathcal{H}_{A} \otimes \mathcal{H}_{B}$. The entanglement entropy is the von Neumann entropy

$$
S(A)=-\operatorname{Tr}\left(\rho_{A} \log \rho_{A}\right)
$$

with

$$
\rho_{A}=\operatorname{Tr}_{B}(|\Psi><\Psi|) .
$$

As $S(A)$ decreases, the area of the minimum surface separating A and B decreases according to the Ryu-Takayanagi prescription which greatly generalises the original Bekenstein-Hawking area formula for the entropy of a black hole. The sphere becomes a dumbbell shape. In the limit $S(A) \rightarrow 0$, the spacetimes $\mathrm{A}$ and $\mathrm{B}$ become classically disconnected.

Let us briefly consider entanglement of two-state quantum mechanical systems, or quantum bits usually shortened to qubits. One qubit can be in the general state

$$
\Psi=\alpha_{\uparrow}\left|\uparrow>+\alpha_{\downarrow}\right| \downarrow>
$$

with probabilities $\left|\alpha_{\uparrow}\right|^{2}$ and $\left|\alpha_{\downarrow}\right|^{2}$ of measuring $\mid \uparrow>$ and $\mid \downarrow>$ respectively.

For two qubits the most general state is

$$
\left|\Psi>=\alpha_{\uparrow \uparrow}\right| \uparrow \uparrow>+\alpha_{\uparrow \downarrow}\left|\uparrow \downarrow>+\alpha_{\downarrow \uparrow}\right| \downarrow \uparrow>+\alpha_{\downarrow \downarrow} \mid \downarrow \downarrow>
$$

with $\Sigma\left|\alpha_{i j}\right|^{2}=1$.

If we measure the first qubit to be $|\uparrow\rangle$, then the normalized post-measurement state is

$$
\mid \Psi>^{\prime}=\left[\frac{\alpha_{\uparrow \uparrow}\left|\uparrow \uparrow>+\alpha_{\uparrow \downarrow}\right| \uparrow \downarrow>}{\sqrt{\left|\alpha_{\uparrow \uparrow}\right|^{2}+\left|\alpha_{\uparrow \downarrow}\right|^{2}}}\right]
$$

The phenomenon of entanglement is clearest in an EPR state

$$
\mid \Psi>=\frac{1}{\sqrt{2}}[|\uparrow \uparrow>+| \downarrow \downarrow>]
$$

named for a paper which tried unsuccessfully to prove that quantum mechanics is incomplete. Eq. (16) is sometimes alternatively called a Bell state for the physicist 
who derived inequalities based on local realism which are violated by quantum mechanics. The correlations which occur in quantum mechanics are stronger than could ever exist between classical systems. The point about the EPR/Bell state Eq. (16) is that measuring the first qubit as $\mid \uparrow>$ implies that measurement of the second qubit must with $100 \%$ certainty result in $|\uparrow\rangle$, a correlation which never happens in classical mechanics.

After these digressions, we return to our central point of the turnaround in cyclic cosmology. The CBE model with its empty contraction era aims to jettison the accrued entropy at the turnaround so that the contracting universe has zero entropy. One reason for this is that the presence of matter including black holes will disallow contraction to the bounce because black holes will merge and enlarge and in the presence of any matter going in reverse through phase transitions will be entropically impossible. Thus the idea was that the causal patch at turnaround which is selected from a very large number of candidates, as suggested by the comparison between the two turnaround radii in Eqs.(3) and (5), will almost always be empty of matter. The tiny fraction which do contain matter will represent failed universes which will experience a premature bounce rather than a successful contraction.

Mathematicians or string theorists may provide details of a non-singular turnaround but until then we remain temporarily satisfied with the phenomenological Eq.(??) with a view first to discover a scenario which can be infinitely cyclic.

As the extroverse spacetime is stretched by the extreme amount in Eq.(3) it will become disconnected into causal patches and this disconnection corresponds precisely to making $S$ (int) vanish where $S($ int $)$ is the entanglement entropy between introverse and extroverse. Because the extroverse becomes a classically disconnected spacetime, we may study the contracting introverse without any further concern for the extroverse whose entropy is thereby jettisoned.

Tolman's work had so much impact that almost no work on cyclic cosmology was pursued thereafter. The most important subsequent step was the discovery of dark energy in 1998 which provides the clear division into introverse and extroverse as discussed in the previous section. Tolman was necessarily considering the entropy for the whole universe and applied the second law of thermodynamics to it, to arrive at his impossibility theorem.

Tolman was certainly aware of quantum mechanics but it surely never entered his head that quantum mechanics is relevant to cosmology. Such an idea only emerged long after Tolman died in 1948, in the 1980s when quantum fluctuations were proposed to seed structure formation. Now we learn that, at the other end of the expansion era, quantum mechanics may play a rôle also in the spacetime disconnection of the introverse, as well as in choosing the quantum entanglement entropy of the introverse to be the quantity which is infinitely cyclic. 
As for experimental tests of CBE cyclic cosmology, regrettably only one is immediately apparent which is the prediction that the equation of state of dark energy is a constant $\omega=-1$ very precisely to many decimal places. If a measurable departure from this prediction were found experimentally, it would refute the CBE cyclic model. Hopefully with more work on the model, other testable predictions will be discovered.

\section{Discussion}

Quantum mechanics was originally discovered as a description for atomic physics with no anticipation that it would be useful in theoretical cosmology. The first such use was several decades later based on quantum perturbations in the early universe seeding the growth of large scale structure; this is well-known in inflationary scenarios and less known in cyclic bounce models.

In the present article we have suggested a second use of quantum mechanics in cosmology. It arose by better understanding the CBE model. The underlying quantum theory is based on AdS/CFT duality and entropic entropy where a source of encouragement is the emergent understanding of AdS/CFT and the Ryu-Takayanagi formula from analysis of holographic quantum error-correcting codes.

It is a decade since publication of $\mathrm{BF}$ which did generate a lot of discussion at that time In addressing Tolman's no-go theorem, the present improved suggestion is to make an infinitely-cyclic cosmology by requiring infinite cyclicity not of the entropy of the whole universe but only of the quantum entanglement entropy of the introverse, $S($ int $)$.

This has the advantage that when $S($ int $)=0$ at turnaround, the introverse becomes a spacetime manifold disconnected from the extroverse and hence the latter can be jettisoned with impunity. This avoids the appearance of an infiniverse after infinitely many cycles and enables a model with only one universe.

\section{Afterword}

Just to add a few final words on a different topic which is the idea that dark matter constituents are PIMBHs(=Primordial Intermediate Mass Black Holes). Our proposal $^{\underline{5}}$ is that the Milky Way contains between ten million and ten billion massive black holes each with between twnety and a hundred thousand times the solar mass. Assuming the halo is a sphere of radius a hundred thousand light years the typical separation is between one hundred and one thousand light years which is also the most probable distance of the nearest PIMBH to the Earth.

Of the detection methods discussed in $\stackrel{5}{ }$, extended microlensing observations seem the most promising and an experiment to detect higher longevity microlensing 
events is being actively pursued. The wide-field telescope must be in the Southern Hemisphere to use the Magellanic Clouds (LMC and SMC) for sources. The best existing (since 1986) telescope is Blanco 4m fitted with the DECam having 520 Megapixels. The future LSST(= Large Synoptic Survey Telescope) under construction in Northern Chile will not take first light until 2022. It will be $8.4 \mathrm{~m}$ with a 3200 Megapixel camera. Long before LSST, at the Blanco $4 \mathrm{~m}$ in Chile, there is an approved NOAO proposal $\underline{6}$ for a microlensing experiment which already started February 2018 and expects results by the end of 2019 .

\subsection{The Reason for Dark Matter}

The question why there is dark matter seems to us to be equally as important as what is the dark matter, and there is more discussion in ${ }^{7}$ The answer to why is the second law of thermodynamics applied to the entropy of the universe. It is not a sharp argument since it depends on dynamics, and to make it rigorous would require solving Boltzmann's equations for every particle in the universe which is impracticable. Nevertheless, it is strongly suggestive and we believe it can be the correct reason. The entropy from the SMBHs at galactic cores gives $S / k \sim 10^{103}$ and the identification $D M=P I M B H s$ can increase this to $S / k \sim 10^{106}$ depending on the PIMBH mass function and the dark matter was formed in the first three years.

\section{References}

1. L. Baum and P.H. Frampton,

Turnaround in Cyclic Cosmology.

Phys. Rev. Lett. 98, 071301 (2007). arXiv:hep-th/0610213

2. R.C. Tolman,

On the Theoretical Requirements for a Periodic Behaviour of the Universe.

Phys. Rev. 38, 1758 (1931).

3. E.P. Verlinde,

On the Origin of Gravity and the Laws of Newton.

JHEP 1104:029 (2011). arXiv:1001.0785[hep-th]

4. P.H. Frampton,

Holographic Entanglement Entropy and Cyclic Cosmology.

Phys. Dark Univ. 20, 28 (2018). arXiv:1707.01092[gr-qc]

5. P.H. Frampton, Searching for Dark Matter Constituents with Many Solar Masses. MPLA A31, 1650093 (2016). arXiv:1510.00400[hep-ph]

G.F. Chapline and P.H. Frampton,

Intermediate Mass MACHOs: A New Direction for Dark Matter Searches.

JCAP 11:042 (2016). $\operatorname{arXiv:1608.04297[gr-qc].~}$

6. W. Kinney, et al., https://www.noao.edu/noaoprop/abstract.mpl?2018A-0273

13702/session/5/contribution/106/material/paper/0.pdf

7. P.H. Frampton,

On the Nature and Origin of Cosmological Dark Matter. 1804.03516 [physics.gen-ph]. 